\title{
REGULARIZATION OF \\ LINEAR OPERATOR EQUATIONS
}

\author{
M. A. LUKas
}

In this thesis, we examine regularization for the approximate solution of a class of bounded linear operator equations $K u=f$, where $f$ need not be given exactly. We are particularly interested in Fredholm integral equations of the first kind and the problem of data smoothing.

In Chapter 2, we consider Tikhonov regularization and regularization with differential operators. Existing results concerning the existence, uniqueness, convergence and construction of the regularized solution are reviewed and extended, and some new results are proved. We also derive the important filtering property of these forms of regularization.

In Chapter 3, we consider regularization with discrete and possibly noisy data $d_{i}=f\left(x_{i}\right)+\varepsilon_{i}, i=1,2, \ldots, n$; that is,

$$
\underset{u \in W}{\operatorname{minimize}} \frac{1}{n} \sum_{i=1}^{n}\left(K u\left(x_{i}\right)-d_{i}\right)^{2}+\alpha \Omega(u)
$$

Here $W=W^{m, 2}[0,1]$ and $\Omega(u)=\|u\|_{W}^{2}$ or $\Omega(u)=\|T u\|_{2}^{2}$, where $T$ is a linear differential operator of order $m$. Two existing methods for constructing the regularized solution $u_{\alpha}$ are described and related.

Up to date most authors have implemented regularization by arbitrarily fixing the order of regularization $m$ and then choosing $\alpha$ by some known method. In Chapter 4, we show that better results are obtained if $m$ is determined first. An optimal value of $m$ is defined in terms of an

Received 16 June 1981. Thesis submitted to the Australian National University January 1981. Degree approved June 1981. Supervisor: Dr R.S. Anderssen. 
optimal filtering criterion obtained by minimizing

$$
\frac{1}{n} E\left\|K_{\alpha, m}-f\right\|^{2}=\frac{1}{n} E \sum_{i=1}^{n}\left(K_{u_{\alpha, m}}\left(x_{i}\right)-f\left(x_{i}\right)\right)^{2}
$$

with respect to $m$ and $\alpha$. Furthermore, a practical estimate of the optimal filtering order is proposed.

In Chapter 5, we define a class of optimal regularization parameters $\alpha_{0, \mu}$, which includes the minimizer $\alpha_{0}$ of $\frac{1}{n} E\left\|K u_{\alpha}-f\right\|^{2}$. We derive some properties of $\alpha_{0, \mu}$, including its aysmptotic behaviour as $n \rightarrow \infty$. Relating earlier work, it is shown that for a reasonable statistical estimate $\beta$ of $\alpha_{0}, \frac{1}{n} E\left\|K_{B, m}-f\right\|^{2}$ should be close to the minimum for the optimal filtering order of regularization $m$. We derive an almost unbiased estimate $\alpha_{\mu}^{\prime}$ of $\alpha_{0, \mu} ;$ in particular, an estimate $\alpha^{\prime}$ of $\alpha_{0}$ and some of its statistical properties. The estimate $\alpha^{\prime}$ is shown to have a strong connection with the weighted cross-validation estimate.

Chapter 6 is concerned with some practical aspects of regularization. Numerical results are presented which demonstrate the viability of the estimates considered in Chapters 4 and 5 .

Department of Mathematics, Colorado State University, Fort Collins, Colorado 80523, USA. 